\title{
A RIEMANN-TYPE INTEGRAL BASED ON THE SECOND SYMMETRIC DERIVATIVE
}

\author{
C. FREILING, D. RINNE AND B. S. THOMSON
}

\begin{abstract}
An integral of the generalized Riemann type is developed which inverts the Schwarz derivative of a continuous function.
\end{abstract}

\section{Introduction}

The second order symmetric derivative of a continuous function $F$ is defined by the expression

$$
\mathrm{SD}_{2} F(x)=\lim _{h \rightarrow 0+} \frac{F(x+h)+F(x-h)-2 F(x)}{h^{2}} .
$$

This is called sometimes the Riemann derivative or often, with less justification, the Schwarz derivative. It arises in Riemann's classical study of trigonometric series and its use there has kept it as an object of study for nearly a century and a half.

The problem we wish to address is the inversion of this derivative. That is, if $\mathrm{SD}_{2} F(x)=f(x)$ everywhere for some continuous function $F$, and $f$ is given, how may $F$ be determined? This problem arises directly from the coefficient problem for trigonometric series: if a trigonometric series

$$
a_{0} / 2+\sum_{n=1}^{\infty} a_{n} \cos n x+b_{n} \sin n x
$$

converges everywhere to a sum $f(x)$, how may the coefficients of the series be determined? Of course if $f$ is Lebesgue integrable then one expects the coefficients to be determined by the usual Fourier formulas using Lebesgue integrals. But such an $f$ need not be integrable in any of the familiar senses.

The first solutions of this problem are by Denjoy [4], Marcinkiewicz and Zygmund [10], James [9] and Burkill [3]. All involve the inversion of the derivative $\mathrm{SD}_{2} G(x)$ or some natural variant.

It is our purpose in this article to present an integral, defined very nearly as a limit of Riemann sums, that inverts the second order symmetric derivative of continuous functions and, hence, that solves the coefficient problem. Since the introduction of the Henstock-Kurzweil integral some three decades ago, a number of researchers (see,

Received 4 October 1993; revised 7 June 1995.

1991 Mathematics Subject Classification 26A24.

Research of the first two authors was supported in part by grants from NSF; research of the third author was supported in part by a grant from NSERC.

J. London Math. Soc. (2) 56 (1997) 539-556 
for example, $[\mathbf{8}, \mathrm{p} . \mathrm{vi}])$ have wondered whether analogous ideas could be applied to these problems. A Riemann-type integral that inverts approximate symmetric derivatives and also solves the coefficient problem has appeared in [11], based on covering ideas originating in [6]. In this article we develop the tools needed for a similar study of the second order symmetric derivative.

\section{Some preliminary definitions}

\subsection{Rectangles and 2-intervals}

Our setting is the real line and the plane. By a rectangle $R$ we mean merely a product of two closed intervals

$$
R=[a, b] \times[c, d] .
$$

The eccentricity of a rectangle $R$ is the maximum ratio of its two sides,

$$
e(R)=\max \left\{\frac{d-c}{b-a}, \frac{b-a}{d-c}\right\} .
$$

If $R=\bigcup_{i=1}^{n} R_{i}$ where $R, R_{1}, R_{2}, \ldots, R_{n}$ are rectangles and the $\left\{R_{i}\right\}$ do not overlap, then we call the finite sequence $\left(R_{1}, R_{2}, \ldots, R_{n}\right)$ a partition of $R$. Any subsequence of $\left(R_{1}, R_{2}, \ldots, R_{n}\right)$ shall be called a subpartition of $R$.

By a 2-interval $K$ we mean an ordered pair of intervals of the form

$$
K=([a, b],[a+p, b-p]) \quad \text { with } a<b, 0<p \leqslant \frac{1}{2}(a+b) .
$$

Note that this includes the possibility that the second interval of the pair is degenerate. The center of the two intervals is the same and we denote it as $c(K)$. For any 2-interval $K$ with center $c$ we have then

$$
K=([c-h, c+h],[c-k, c+k])
$$

for some $0 \leqslant k<h$. We write

$$
|K|=h \quad \text { and } \quad\|K\|=h^{2}-k^{2}
$$

and refer, loosely, to $|K|$ as the length of $K$ and to $\|K\|$ as the area of $K$. We refer to $c \pm h$ as the outer endpoints of $K$ and $c \pm k$ as the inner endpoints of $K$. The 2-interval

$K$ is said to be $r$-regular if $0 \leqslant k \leqslant r h$. For all of our applications $r=\frac{1}{3}$ and so the condition will read $0 \leqslant 3 k \leqslant h$.

Every 2 -interval $K$ may be regarded as the 'projection' of a rectangle $R$ by the following scheme. Write $\pi:(x, y) \rightarrow(x+y)$ for the projection from $\mathbb{R}^{2}$ to $\mathbb{R}$. Then, if $R=[a, b] \times[c, d]$ is a rectangle, the four corners project to the four points $\pi(a, c)$, $\pi(b, c), \pi(b, d)$ and $\pi(a, d)$, which can be viewed as the four vertices of a 2-interval. The points $\pi(a, c)$ and $\pi(b, d)$ form its outer endpoints and the points $\pi(b, c)$ and $\pi(a, d)$ its inner endpoints. We write $\pi([a, b] \times[c, d])$ for the 2 -interval. For example, if $b+c<a+d$, then

$$
\pi([a, b] \times[c, d])=([a+c, b+d][b+c, a+d]) .
$$

Naturally there is a close connection between properties of the rectangle $R$ and its associated 2-interval $\pi(R)$. Notice that the center of the 2-interval is given by

$$
c(\pi([a, b] \times[c, d]))=\frac{1}{2}(a+b+c+d) .
$$




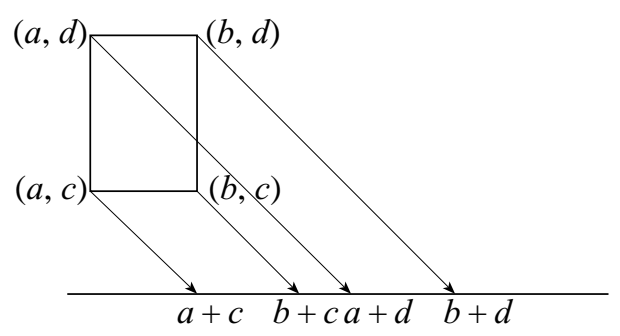

FIG. 1. A rectangle projecting to a 2-interval.

The area of the 2-interval is

$$
\begin{aligned}
\|\pi([a, b] \times[c, d])\| & =\frac{1}{4}(b+d-a-c)^{2}-\frac{1}{4}(a+d-b-c)^{2} \\
& =b d-b c-a d+a c=(b-a)(d-c)
\end{aligned}
$$

which latter is exactly the area of the rectangle. Similarly the length of a 2 -interval is

$$
|\pi([a, b] \times[c, d])|=\frac{1}{2}(b+d-a-c),
$$

which is one quarter of the perimeter of the rectangle. The regularity of a 2-interval is related to the eccentricity of a rectangle that projects to it; in particular, if $R$ has eccentricity no more than $e$ then $\pi(r)$ is $(e-1) /(e+1)$-regular. We mainly require $\frac{1}{3}$-regularity and this holds for $e \leqslant 2$.

Given any 2-interval $K$ we define a partition into 'smaller' 2-intervals by means of the projections. If $R$ is any rectangle with $\pi(R)=K$ and $\left(R_{1}, R_{2}, \ldots, R_{n}\right)$ is a partition of $R$ then we shall call

$$
\left(\pi\left(R_{1}\right), \pi\left(R_{2}\right), \ldots, \pi\left(R_{n}\right)\right)
$$

a 2-partition of the 2-interval $K$. If $\left(R_{1}, R_{2}, \ldots, R_{n}\right)$ is a subpartition of $R$ then we shall call (8) a 2-subpartition of $K$. Thus a finite sequence $\left(K_{1}, K_{2}, \ldots, K_{n}\right)$ of 2 -intervals is a 2-partition or 2-subpartition of $K$ if it can be so expressed. While the members of the sequence $\left(R_{1}, R_{2}, \ldots, R_{n}\right)$ must be distinct in order for them to form a partition of $R$, there can easily be duplications in the sequence (8). We also need to be able to refer to the position of a member in the sequence; for these reasons we must use the language of sequences here rather than sets.

Let $\delta$ be a gauge (that is, a positive function) defined on $(a, b)$, let

$$
K=([a, b],[a+p, b-p])
$$

be a 2-interval and suppose that $\left(K_{1}, K_{2}, \ldots, K_{n}\right)$ is a 2-partition or a 2-subpartition of $K$; we say that it is $\delta$-fine at the centers if for each $i=1,2, \ldots, n$ we have

$$
\left|K_{i}\right|<\delta\left(c\left(K_{i}\right)\right) \text { for } i=1,2, \ldots, n .
$$

We need occasionally to measure the fineness of a partition at an outer endpoint of a 2-interval; let us say that a 2 -interval $K_{i}$ is $\delta$-fine at the endpoint $a_{i}$ if $\left|K_{i}\right|<\delta\left(a_{i}\right)$. The partition is $r$-regular if each member $K_{i}$ is $r$-regular.

\subsection{Functions on rectangles and 2-intervals}

All functions that appear are real-valued functions defined on the real line, the plane or defined on rectangles or 2-intervals. 
Let $F$ be a real function defined on $\mathbb{R}$. The expression

$$
\Delta_{s}^{2} F(x, h)=F(x+h)+F(x-h)-2 F(x)
$$

is called the second order symmetric difference of $F$ at $x$. Most of our concerns in this article arise from this difference. We recall some of the terminology that has evolved. A function $F$ is said to be smooth at a point $x$ if $\Delta_{s}^{2} F(x, h)=o(h)$ as $h \rightarrow 0+$. For an arbitrary function $F$ the extreme second order symmetric derivatives are defined as

$$
\overline{\mathrm{SD}}_{2} F(x)=\limsup _{h \rightarrow 0+} \frac{\Delta_{s}^{2} F(x, h)}{h^{2}} \text { and } \quad \underline{\mathrm{SD}}_{2} F(x)=\liminf _{h \rightarrow 0+} \frac{\Delta_{s}^{2} F(x, h)}{h^{2}} .
$$

If these are equal and are finite we write their common value as $\mathrm{SD}_{2} F(x)$, which is called the second order symmetric derivative; it is this derivative to which the title of the paper refers and which serves as the underlying basis for the integral that is to be developed.

There is an evident connection with 2-intervals obtained by associating the 2interval $([x-h, x+h],[x, x])$ with this expression. The connection with arbitrary $r$ regular 2 -intervals will come about because of the following elementary computation: if $0 \leqslant k \leqslant r h$, then

$$
h^{2}+k^{2} \leqslant\left(1+r^{2}\right) h^{2}=\frac{1+r^{2}}{1-r^{2}}\left(h^{2}-r^{2} h^{2}\right) \leqslant \frac{1+r^{2}}{1-r^{2}}\left(h^{2}-k^{2}\right) .
$$

We shall use the notation

$$
C_{r}=\frac{1+r^{2}}{1-r^{2}}
$$

Throughout the article the only regularity condition imposed will be the use of $\frac{1}{3}$-regular 2-intervals and so $r=\frac{1}{3}$ and $C_{1 / 3}=\frac{10}{8}$.

By definition the existence of the derivative $\mathrm{SD}_{2} G(x)=f(x)$ requires that for every $\epsilon>0$ there is a $\delta>0$ so that

$$
\left|G(x+h)+G(x-h)-2 G(x)-f(x) h^{2}\right|<\epsilon h^{2}
$$

for $0<h<\delta$. Now we see easily, using (10) and (11), that this translates into the requirement that

for $0 \leqslant 3 k \leqslant h<\delta$.

$$
\begin{gathered}
\mid G(x+h)+G(x-h)-G(x+k)-G(x-k))-f(x)\left(h^{2}-k^{2}\right) \mid \\
<\epsilon\left(h^{2}+k^{2}\right) \leqslant \epsilon C_{1 / 3}\left(h^{2}-k^{2}\right)
\end{gathered}
$$

We can translate this into the language of 2-interval functions. For any 2-interval $K=([c-h, c+h],[c-k, c+k])$ and any real function $G$ we define

$$
\Delta_{K} G=G(c-h)+G(c+h)-G(c-k)-G(c+k) .
$$

Now writing $K=([x-h, x+h],[x-k, x+k])$ and using (12) the inequality above translates directly to the statement that

$$
\left|\Delta_{K} G-f(x)\|K\|\right|<\epsilon C_{1 / 3}\|K\|
$$

for $x=c(K),|K|<\delta$ provided that $K$ is $\frac{1}{3}$-regular.

For any rectangle $R=[a, b] \times[c, d]$ and any function $H$ of two variables we write the difference

$$
H(R)=H(a, c)-H(a, d)-H(b, c)+H(b, d) .
$$


In particular note that if the sequence $\left(R_{1}, R_{2}, \ldots, R_{n}\right)$ is a partition of $R$ then

$$
H(R)=\sum_{i=1}^{n} H\left(R_{i}\right)
$$

so that $H$ is interpreted as an additive rectangle function.

If we write $H(x, y)=G(x+y)$, then notice that

$$
H(R)=\Delta_{\pi(R)} G
$$

for any rectangle $R$, thus linking (14) and (12). As a result of this we see, using (15), that if the sequence $\left(K_{1}, K_{2}, \ldots, K_{n}\right)$ is a 2-partition of the 2-interval $K$ then

$$
\Delta_{K} G=\sum_{i=1}^{n} \Delta_{K_{i}} G
$$

\section{Covering theorems}

The integral will be defined as a certain limit of Riemann sums taken over 2partitions. Justification for the integral requires that in the setting of the definition such 2-partitions necessarily exist. This is supplied by covering theorems which are modifications of one from [1].

In the first theorem (Theorem 8) we obtain the most refined version with an explicitly determined exceptional set that is 'splattered'. This version requires strong assumptions on the gauge that we wish to avoid in defining the integral. The second version (Theorem 9) is the one we use throughout and is the form useful to us. Note that the term gauge means merely a positive function; this is the most common language currently in use in discussions of Riemann-type integrals.

In order to define an appropriate integral it seems we would be required to show that for any gauge $\delta$ on $\mathbb{R}$ and any 2-interval $K$ there is a sequence $\left(I_{1}, I_{2}, \ldots, I_{n}\right)$ forming a 2-partition of the $K$ that is $\frac{1}{3}$-regular and such that each $I_{i}$ is $\delta$-fine at the center. This cannot be exactly true: if it were one could conclude that any function $G$ that satisfies $G(x+h)+G(x-h)-2 G(x)=0$ locally satisfies that identity globally, and this is false (for example, let $G(x)=\operatorname{sgn}(x)$ ). The form of the covering theorem thus needs a modification allowing extra members of the partition that are not $\delta$-fine. We show that there is a countable set $E=\left\{x_{1}, x_{2}, x_{3}, \ldots\right\}$ with the property: for any 2-interval

$$
K=([a, b],[a+p, b-p])
$$

and for any gauge $\gamma$ on $\mathbb{N} \times \mathbb{N}$ there is a sequence

$$
\left(I_{1}, I_{2}, \ldots, I_{n}\right)
$$

forming a 2-partition of the 2-interval $K$ that is $\frac{1}{3}$-regular, such that each $I_{i}$ is either $\delta$-fine at the center or else $I_{i}$ has an outer endpoint $x_{j}$ in $E$ with $\left|I_{i}\right|<\gamma(i, j)$.

The lemmas which now follow develop the machinery for such a proof.

\subsection{Geometrical arguments}

Lemma 1. Let $\delta$ be a gauge in the plane which is constant on lines with slope -1 . Let $L$ be the union of a closed set of lines with slope -1 and let $D$ denote the complement of $L$. Suppose that $L^{\prime}$ is a union of some subcollection of the lines in $L$ such that $L^{\prime}$ is 
dense in $L$. Then, for any $a<b$ and any $y$ there is a c such that $\frac{1}{2}(b-a) \leqslant c-y \leqslant 2(b-a)$ and the rectangle $R=[a, b] \times[y, c]$ can be partitioned into subrectangles such that each rectangle in the partition is of one of the following types:

$(\alpha)$ its center is in $L^{\prime}$ and its eccentricity is less than or equal to 2 ,

$(\beta)$ its interior is in the open set $D$,

$(\gamma)$ it is a $\delta$-fine square with center in $L$.

Proof. We may assume without loss of generality that $a=0, b=1$ and that $y=0$.

Case 1. Some line $l$ in $L$ (and hence also then some line $l^{\prime}$ in $L^{\prime}$ ) intersects the $y$-axis in the interval $\left(\frac{3}{4}, \frac{3}{2}\right)$ (see Figure 2).

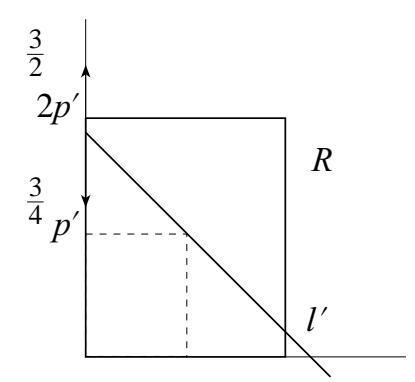

FIG. 2. Case 1 in Lemma 1.

Then $l^{\prime}$ also intersects the vertical line $x=\frac{1}{2}$ at some point $\left(\frac{1}{2}, p^{\prime}\right)$ where $\frac{1}{4}<p^{\prime}<1$. Then if we let $c=2 p^{\prime}$ the rectangle $R=[0,1] \times[0, c]$ has center $\left(\frac{1}{2}, p^{\prime}\right)$ and is of type $\alpha$ so we are done.

For the remaining cases we shall take $c=\frac{1}{2}$ so that $R=[0,1] \times\left[0, \frac{1}{2}\right]$.

Case 2. Some line $l$ in $L$ intersects the $y$-axis in the interval $\left(\frac{1}{2}, \frac{3}{4}\right]$ but not in $\left(\frac{3}{4}, \frac{3}{2}\right)$ as for Case 1 . Let $l$ be that line in $L$ with the greatest $y$-intercept in $\left(\frac{1}{2}, \frac{3}{4}\right]$. Since $l$ has a $y$-intercept exceeding $\frac{1}{2}$ it must intersect the horizontal line $y=\frac{1}{4}$ at some point $\left(p, \frac{1}{4}\right)$ where $\frac{1}{4}<p \leqslant \frac{1}{2}$ (see Figure 3 ). Then, since $L^{\prime}$ is dense in $L$, there is a line

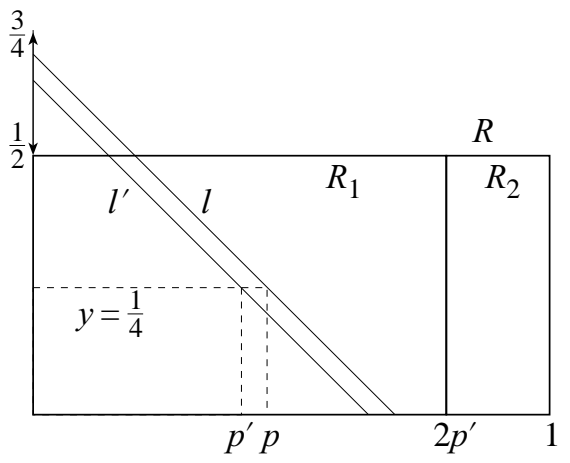

FIG. 3. Case 2 in Lemma 1.

$l^{\prime}$ in $L^{\prime}$ that is sufficiently close to $l$ that it intersects the horizontal line $y=\frac{1}{4}$ at some point $\left(p^{\prime}, \frac{1}{4}\right)$ where $\frac{1}{2} p+\frac{1}{8}<p^{\prime} \leqslant p$. Note that $2 p^{\prime}>p+\frac{1}{4}$ which is the $x$-intercept 
of $l$. Thus $R$ can be partitioned into the two rectangles $R_{1}=\left[0,2 p^{\prime}\right] \times\left[0, \frac{1}{2}\right]$ and $R_{2}=\left[2 p^{\prime}, 1\right] \times\left[0, \frac{1}{2}\right]$ with $R_{1}$ of type $(\alpha)$ since its center is on the line $l^{\prime}$ and $R_{2}$ of type $(\beta)$ by the assumptions on $L$ and the maximality of $l$.

Case 3. Some line $l$ in $L$ intersects the $y$-axis in the interval $\left(0, \frac{1}{2}\right]$ but not in $\left(\frac{1}{2}, \frac{3}{2}\right)$ as for Cases 1 and 2 . Let $l$ be that line in $L$ with the greatest $y$-intercept $(0, p)$ in $\left(0, \frac{1}{2}\right]$. We may choose $l^{\prime}$ in $L^{\prime}$ so that $l^{\prime}$ has $y$-intercept $\left(0, p^{\prime}\right)$ with $p-p^{\prime}$ as small as we please (see Figure 4).

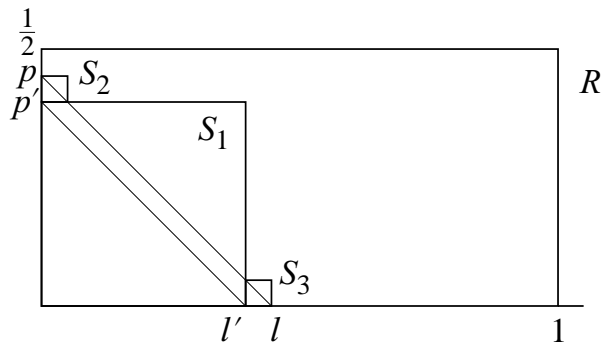

Fig. 4. Case 3 in Lemma 1.

Note that for any choice of $l^{\prime}$ the square $S_{1}=\left[0, p^{\prime}\right] \times\left[0, p^{\prime}\right]$ is of type $(\alpha)$, while the squares $S_{2}=\left[0, p-p^{\prime}\right] \times\left[p^{\prime}, p\right]$ and $S_{3}=\left[p^{\prime}, p\right] \times\left[0, p-p^{\prime}\right]$ have centers on the line $l$. (If $p=p^{\prime}$ then the squares are degenerate and can be left out.) The remainder of the rectangle, that is, $R \backslash\left(S_{1} \cup S_{2} \cup S_{3}\right)$, has its interior in $D$ and so can be partitioned into subintervals of type $(\beta)$. We have then only to choose $p^{\prime}$ sufficiently close to $p$ so that squares $S_{2}$ and $S_{3}$ are $\delta$-fine at their centers (recall that $\delta$ is constant on $l$ ). Accordingly we have partitioned $R$ as required.

Case 4. In the final case no line $l$ in $L$ intersects the $y$-axis in the interval $\left(0, \frac{3}{2}\right)$. Then $L$ does not meet the interior of the rectangle $R$ and so $R$ itself if of type $(\beta)$ and we are done.

Lemma 2. Let $\delta, L, L^{\prime}$ and $D$ be as in the preceding lemma and let $S$ denote the square $[a, b] \times[a, b]$. Then there is a subrectangle $R=[a, d] \times[a, b]$ of $S$ where $d-a \geqslant \frac{1}{12}(b-a)$ and $R$ can be partitioned into rectangles each of type $(\alpha),(\beta)$ or $(\gamma)$.

Proof. As before we may assume that $a=0$ and $b=1$ so that $S$ is the unit square $[0,1] \times[0,1]$.

Case 1. There is a line $l$ in $L$ (and hence also a line $l^{\prime}$ in $L^{\prime}$ ) such that its $y$-intercept is in $\left(\frac{3}{4}, 1\right)$ (see Figure 5). Such an $l^{\prime}$ intersects the horizontal line $y=\frac{1}{2}$ at some point $\left(p^{\prime}, \frac{1}{2}\right)$ where $\frac{1}{4}<p^{\prime}<\frac{1}{2}$. Then the rectangle $R=\left[0,2 p^{\prime}\right] \times[0,1]$ is of type $(\alpha)$ with center $\left(p^{\prime}, \frac{1}{2}\right)$ in $L^{\prime}$ and $2 p^{\prime} \geqslant \frac{1}{12}$ so that $R$ satisfies the required conditions.

Case 2. Suppose that $L$ does not intersect the $y$-axis between $\frac{3}{4}$ and 1 (see Figure 6). Let $d=\frac{1}{12}$ so that $R$ is the rectangle $\left[0, \frac{1}{12}\right] \times[0,1]$. By the preceding lemma there is a partition of the desired type for the rectangle $R_{1}=\left[0, \frac{1}{12}\right] \times\left[0, c_{1}\right]$ for some choice of $\frac{1}{24} \leqslant c_{1} \leqslant \frac{1}{6}$. Applying the lemma again we partition $R_{2}=\left[0, \frac{1}{12}\right] \times\left[c_{1}, c_{2}\right]$ for some choice of $\frac{1}{24} \leqslant c_{2}-c_{1} \leqslant \frac{1}{6}$. Continue inductively partitioning the interval 


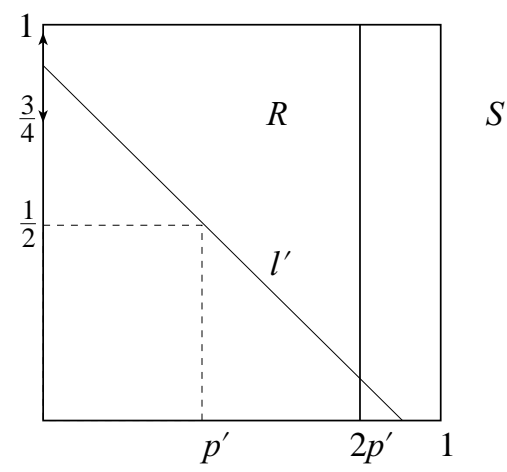

FIG. 5. Case 1 in Lemma 2.

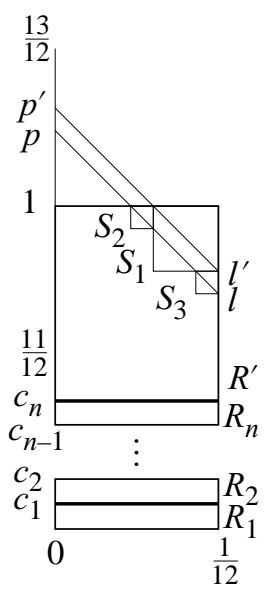

FIG. 6. Case 2 in Lemma 2.

$R_{i}=\left[0, \frac{1}{12}\right] \times\left[c_{i-1}, c_{i}\right]$ for some choice of $\frac{1}{24} \leqslant c_{i}-c_{i-1} \leqslant \frac{1}{6}$ until some $c_{n} \in\left[\frac{3}{4}, \frac{11}{12}\right]$. This must occur at some stage since $\frac{11}{12}-\frac{3}{4}=\frac{1}{6}$. In this way we have obtained a partition of the required form for the rectangle

$$
\left[0, \frac{1}{12}\right] \times\left[0, c_{n}\right]=\bigcup_{i=1}^{n} R_{i} .
$$

We shall be done if we can also partition the rectangle $R^{\prime}=\left[0, \frac{1}{12}\right] \times\left[c_{n}, 1\right]$.

If $R^{\prime}$ has its interior entirely in $D$ then immediately $R^{\prime}$ itself is of type $(\beta)$ and we are done. If not then there is at least one line $l$ in $L$ which intersects the $y$-axis at a point $(0, p)$ where $1 \leqslant p<\frac{13}{12}$.

Let $l$ be that line in $L$ with the least $y$-intercept $(0, p)$ in $\left[1, \frac{13}{12}\right)$. We may choose $l^{\prime}$ in $L^{\prime}$ so that $l^{\prime}$ has $y$-intercept $\left(0, p^{\prime}\right)$ with $p^{\prime}-p$ as small as we please.

Note that for any choice of $l^{\prime}$ the square $S_{1}=\left[p^{\prime}-1, \frac{1}{12}\right] \times\left[p^{\prime}-\frac{1}{12}, 1\right]$ is of type $(\alpha)$, while the squares

$$
S_{2}=\left[p-1, p^{\prime}-1\right] \times\left[1-p^{\prime}+p, 1\right] \quad \text { and } \quad S_{3}=\left[\frac{1}{12}-p^{\prime}+p, \frac{1}{12}\right] \times\left[p-\frac{1}{12}, p^{\prime}-\frac{1}{12}\right]
$$

have centers on the line $l$. (Again see Figure 6; this argument is a repetition of the ideas found in Case 3 of the preceding lemma. Note that if $p=p^{\prime}$ then the squares $S_{2}$ and $S_{3}$ are degenerate and can be left out.) 
The remainder of the rectangle, that is, $R^{\prime} \backslash\left(S_{1} \cup S_{2} \cup S_{3}\right)$, has its interior in $D$ and so can be partitioned into subintervals of type $(\beta)$. We have only then to choose $p^{\prime}$ sufficiently close to $p$ so that squares $S_{2}$ and $S_{3}$ are $\delta$-fine at their centers (recall that $\delta$ is constant on $l$ ). Accordingly, we have partitioned $R^{\prime}$ and hence also $R$ as required.

Lemma 3. Let $\delta, L, L^{\prime}$ and $D$ be as in the preceding lemmas. Let $R$ be any rectangle, $x$ any point inside $\pi(R)$ and $N$ any neighborhood of $x$. Then $R$ can be partitioned into rectangles, each of type $(\alpha),(\beta)$ or $(\gamma)$, together with up to four rectangles with eccentricity not exceeding 2 that project into $N$ and have an outer corner that projects to $x$.

Proof. Quadrasect $R$ by a horizontal line and a vertical line so that $P$, the common vertex of the rectangles, projects to $x$. We show that each of these four can be partitioned into rectangles, each of type $(\alpha),(\beta)$ or $(\gamma)$, together with one further rectangle, with eccentricity not exceeding 2 , that projects into $N$ and has an outer corner that projects to $x$. The lemma then follows.

Without loss of generality we may assume that $|N|<\delta((x, 0))$ if $x \in \pi(L)$, and $N \subset \pi(D)$ if $x \in \pi(D)$.

We illustrate the arguments with the $T$ chosen as that subrectangle with lower right corner $P$ (that is, $T$ is the northwest rectangle). The southeast rectangle admits an identical treatment and the remaining two rectangles (northeast and southwest) are slightly easier.

Let $S_{1}$ denote the largest square contained in $T$ and containing the corner of $T$ that is opposite to $P$. If $S_{1}$ contains the left edge of $T$ we can, by applying Lemma 2, obtain a partition of a rectangle $T_{1} \subset S_{1}$ where $T_{1}$ contains at least the left $\frac{1}{12}$ th of $S_{1}$. If $S_{1}$ contains the top edge of $T$ we can similarly obtain a partition of a rectangle $T_{1} \subset S_{1}$, where $T_{1}$ contains at least the top $\frac{1}{12}$ th of $S_{1}$. After choosing $T_{1}$ we let $S_{2}$ be the largest square contained in the rectangle $\overline{T \backslash T_{1}}$ again containing the opposite corner to $P$. By the same procedure there is subrectangle $T_{2} \subset S_{2}$ where $T_{2}$ contains at least the left or the top $\frac{1}{12}$ th of $S_{2}$ (see Figure 7).

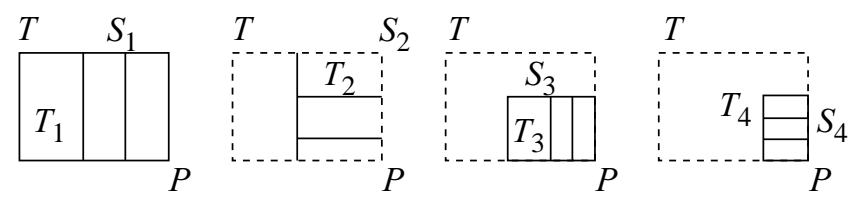

FIG. 7. Partition of $T$ in Lemma 3.

Continuing in this manner we get a sequence of rectangles $T_{1}, T_{2}, T_{3}, \ldots$ each of which may be partitioned as required. At some stage

$$
T^{\prime}=\overline{T \backslash\left(T_{1} \cup T_{2} \cup \ldots T_{n}\right)}
$$

is a rectangle with eccentricity not more than 2 , with a lower right corner $P$, and that projects entirely into the neighbourhood $N$. Note that in this case $P$ is an inner corner of $T^{\prime}$ and so we are not done. However had the original rectangle $T$ been chosen as the northeast or southwest rectangle then this same argument can terminate since $P$ would, in those cases, be an outer corner of $T^{\prime}$ and so $T^{\prime}$ is of the type we require and thus a partition of $T$ itself of the desired type is obtained.

We continue then. Let $S^{\prime}$ be the largest square contained in $T^{\prime}$ and with corner $P$. Note that the center of the square also projects to $x$. If $x \in \pi(L)$ then $S^{\prime}$ is of type 
$(\gamma)$, since $|N|<\delta(x)$, while if $x \in \pi(D) S^{\prime}$ is of type $(\beta)$, since $N \subset \pi(D)$. The remainder of the rectangle $T^{\prime}, T^{\prime} \backslash S^{\prime}$ now has an outer corner projecting to $x$ and can be treated as either a northeast or a southwest rectangle which we already know how to partition in the manner required.

Corollary 4. Let $\delta, L, L^{\prime}$ and $D$ be as in the preceding lemmas. Let $R$ be any rectangle which intersects the open set $D$. Then $R$ can be partitioned into rectangles each of type $(\alpha),(\beta)$ or $(\gamma)$.

Proof. Apply Lemma 3 with $P$ any point in the rectangle that lies in $D$. Then the four subrectangles of the lemma that have corners at $P$ can be chosen inside $D$ and so are of type $(\beta)$.

\subsection{Category arguments}

Lemma 5. Let $L^{\prime}$ be the union of a set of lines with slope -1 . Let $R$ be a rectangle such that $L^{\prime}$ is dense in $R$ and is co-meager in some neighborhood inside $R$. Then $R$ can be partitioned into rectangles of type $(\alpha)$.

Proof. Let $P$ be a point interior to $R$ such that in a neighborhood $N$ of $P$ the family $L^{\prime}$ is co-meager. Quadrasect $R$ by a horizontal line and a vertical line so that $P$ is the common corner of the four resulting rectangles. It will suffice to show that one of these four can be partitioned as described, as symmetric arguments apply to the others. Let $T$ be that subrectangle with lower right corner $P$ and let $S_{1}$ denote the largest square contained in $T$ and containing the corner of $T$ that is opposite to $P$.

If $S_{1}$ contains the left edge of $T$ we can select a rectangle $T_{1} \subset S_{1}$ with center in $L^{\prime}$, where $T_{1}$ contains at least the left half of $S_{1}$. If $S_{1}$ contains the top edge of $T$ we can similarly obtain a rectangle $T_{1} \subset S_{1}$, again with center in $L^{\prime}$, where $T_{1}$ contains at least the top half of $S_{1}$. After choosing $T_{1}$, we let $S_{2}$ be the largest square contained in the rectangle $\overline{T \backslash T_{1}}$, again containing the opposite corner to $P$. By the same procedure there is subrectangle $T_{2} \subset S_{2}$, where $T_{2}$ contains at least the left or the top half of $S_{2}$ and the center of $T_{2}$ is in $L^{\prime}$.

Continuing in this manner we get a sequence of rectangles $T_{1}, T_{2}, T_{3}, \ldots$ each of which has center in $L^{\prime}$ and each with eccentricity no greater than 2 . At some stage

$$
T^{\prime}=\overline{T \backslash\left(T_{1} \cup T_{2} \cup \ldots T_{n}\right)}
$$

is a rectangle with a lower right corner $P$ that lies entirely in the neighborhood $N$. Next we partition $T^{\prime}$ into smaller rectangles each having a length bigger than its height but less than twice the height. It remains to partition each of these smaller rectangles into rectangles of type $(\alpha)$.

Let $T^{\prime \prime}$ be one of these rectangles. We may assume, without loss of generality, that $T^{\prime \prime}=[0, d] \times[0,1]$, where $1<d<2$, and that the square $[0, d]^{2}$ lies entirely in the neighborhood $N$. Write

$$
A=\left\{2 s:\left(s, \frac{1}{2}\right) \in l^{\prime} \text { for some } l^{\prime} \in L^{\prime}\right\} .
$$

Because $L^{\prime}$ is co-meager in $N$, the set $A$ of real numbers is co-meager in the interval $(0,2 d)$. Select a point $t$ in the intersection

$$
A \cap(A-d) \cap\left(\frac{1}{2}, d-\frac{1}{2}\right) .
$$




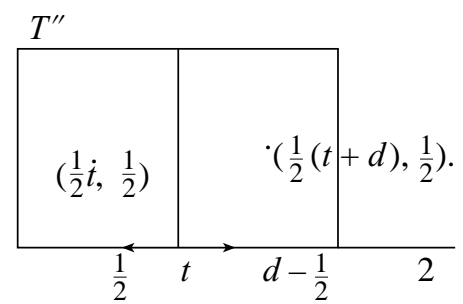

FIG. 8. Partition of $T^{\prime \prime}$ in Lemma 5.

Then the rectangles $[0, t] \times[0,1]$ and $[t, d] \times[0,1]$ (see Figure 8) have centers $\left(\left(\frac{1}{2} t, \frac{1}{2}\right)\right.$ and $\left(\frac{1}{2}(t+d), \frac{1}{2}\right)$ respectively) that lie in $L^{\prime}$. Since $t$ and $d-t$ both lie between $\frac{1}{2}$ and $\frac{3}{2}$ the eccentricity of these rectangles does not exceed 2 as required.

We immediately obtain the following from Lemma 5 and Corollary 4.

Corollary 6. Let $\delta, L, L^{\prime}$ and $D$ be as in the statement of Lemma 1 and suppose that $L^{\prime}$ is co-meager in $L$. Then any rectangle $R$ can be partitioned into rectangles each of type $(\alpha),(\beta)$ or $(\gamma)$.

For convenience we state the Baire category theorem in its splattered form (see [7]). Recall that a set is scattered (clairsemé) if every nonempty subset has an isolated point. A set is right [left] scattered if every nonempty subset has a point isolated on the right [left]. A set is splattered if it may be expressed as the union of a right scattered set and a left scattered set.

Lemma 7 (Splattered Baire). Let $A$ be an open interval of real numbers and suppose that $A=\bigcup_{i=1}^{\infty} A_{i}$ where $A_{1} \subset A_{2} \subset A_{3} \ldots$ Then there is a sequence, possibly transfinite, of open sets $G_{0} \subset G_{1} \subset G_{2} \ldots$ such that

(i) $G_{0}=\varnothing$,

(ii) $G_{\xi}=A$ for some countable ordinal $\xi$,

(iii) if $\lambda$ is a limit ordinal then $G_{\lambda}=\bigcup_{\eta<\lambda} G_{\eta}$,

(iv) for each ordinal $\eta$ there is a set $A_{i}$ dense in $G_{\eta+1} \backslash G_{\eta}$.

The boundary points of the open sets form a splattered set. If the sets $A_{i}$ have the Baire property then 'dense' in (iv) may be replaced by 'co-meager'.

Proof. Assertions (i) and (iii) define the sequence initially and at limit ordinals. Otherwise choose, by the Baire category theorem, an $A_{i}$ which is somewhere dense in $A \backslash G_{\eta}$, say in an interval $I$, and set $G_{\eta+1}=G_{\eta} \cup I$. (If the $A_{i}$ have the Baire property then $I$ and $A_{i}$ may be selected so that, in addition, $\left(I \backslash G_{\eta}\right) \backslash A_{i}$ is meager.) It is clear that the set of right [left] endpoints of the $G_{\eta}$ is left [right] scattered. Finally, by the Cantor-Baire stationary principle, the sequence of open sets will cover $A$ at some countable ordinal.

\subsection{The main covering theorems}

We now state and prove the first covering theorem. 
THEOREM 8. Let $\delta$ be a gauge on $\mathbb{R}$ with the Baire property. Then there is a splattered set $E=\left\{x_{1}, x_{2}, x_{3}, \ldots\right\}$ with the following property: for any 2-interval

$$
K=([a, b],[a+p, b-p])
$$

and for any gauge $\gamma$ on $\mathbb{N} \times \mathbb{N}$ there is a sequence

$$
\left(I_{1}, I_{2}, \ldots, I_{n}\right)
$$

forming a 2-partition of the 2-interval $K$ that is $\frac{1}{3}$-regular, such that each $I_{i}$ is either $\delta$-fine at the center or else has an outer endpoint $x_{j}$ in $E$ with $\left|I_{i}\right|<\gamma(i, j)$.

Proof. For each natural number $i$ let $A_{i}=\left\{x: \delta(x)>i^{-1}\right\}$ and apply the splattered Baire category theorem, Lemma 7 , to obtain a transfinite sequence of open sets $\left\{G_{\eta}\right\}$ with the properties stated. Note that because $\delta$ has the Baire property the condition (iv) of Lemma 7 is met with 'co-meager' rather than merely dense.

It is more convenient to translate the theorem to a statement about rectangles. Let $\delta^{\prime}(x, y)=\delta(x+y)$; then $\delta^{\prime}$ is a gauge on $\mathbb{R}^{2}$ that is constant on lines with slope -1 (so that the lemmas of Sections 3.1 and 3.2 may be applied). Let $E=\left\{x_{1}, x_{2}, x_{3}, \ldots\right\}$ be the splattered set of the endpoints of the $\left\{G_{\eta}\right\}$. We shall show that any rectangle $R$ that projects to a subset of a set $G_{\eta}$ has the following property:

(*) for any gauge $\gamma$ on $\mathbb{N} \times \mathbb{N}$ there is a sequence

$$
\left(R_{1}, R_{2}, \ldots, R_{n}\right)
$$

of subrectangles forming a partition of $R$ such that, for each $i=1,2 \ldots n$, the rectangle $R_{i}$ has eccentricity no more than 2 and either

(i) $R_{i}$ is $\delta^{\prime}$-fine at its center, or

(ii) $R_{i}$ has an outer corner that projects to a point $x_{j}$ in $E$ with $\left|\pi\left(R_{i}\right)\right|<\gamma(i, j)$.

Since $G_{\eta}=\mathbb{R}$ at some stage this proves the theorem.

The property $(*)$ can be more conveniently stated in an equivalent form:

(**) for any gauge $\gamma$ on $\mathbb{N} \times \mathbb{N}$ there is a sequence

$$
\left(R_{1}, R_{2}, \ldots, R_{n}\right)
$$

of subrectangles forming a partition of $R$ such that, for each $i=1,2 \ldots n$, the rectangle $R_{i}$ has eccentricity no more than 2 and either

(i) $R_{i}$ is $\delta^{\prime}$-fine at its center, or

(ii) $R_{i}$ has an outer corner that projects to a point $x_{j}$ in $E$ with $\left|\pi\left(R_{i}\right)\right|<\gamma(i, j)$, or

(iii) $R_{i}$ itself satisfies (*).

We prove that $(*)$ and $(* *)$ are equivalent. It is clear that $\left(^{*}\right) \Rightarrow(* *)$. Conversely suppose $R$ satisfies $(* *)$ and $\gamma$ is a gauge on $\mathbb{N} \times \mathbb{N}$. Then there is a sequence $\left(R_{1}, R_{2}, \ldots, R_{n}\right)$ of subrectangles forming a partition of $R$ such that each $R_{i}$ has eccentricity no more than 2 and each is of type (i), (ii) or (iii). We show how to construct a new sequence $\left(R_{1}^{\prime}, R_{2}^{\prime}, \ldots, R_{m}^{\prime}\right)$ forming a partition of $R$ with each $R_{i}^{\prime}$ having eccentricity no more than 2 and each of type (i) or (ii). It will follow that $R$ satisfies $(*)$ and we are done. Each $R_{i}$ in the original sequence is treated, in turn, in the following manner. If $R_{i}$ is of type (i) or (ii) then set $R_{i}^{\prime}=R_{i}$. If $R_{i}$ is of type (iii) then choose an appropriate gauge $\gamma_{i}$ and apply (*) with that gauge to obtain a partition $\left(R_{i 1}, R_{i 2}, \ldots, R_{i n_{i}}\right)$ of $R_{i}$. 
Replace $R_{i}^{\prime}=R_{i 1}$ and place $\left(R_{i 2}, R_{i 3}, \ldots, R_{i n_{i}}\right)$ at the end of the sequence so far obtained. It is easy to select each $\gamma_{i}$ so that the resulting sequence satisfies (ii) with respect to the original gauge $\gamma$.

Now we observe some facts about $(*)$ (or equivalently $(* *)$ ). The property $(*)$ is additive. If $R=\bigcup_{i=1}^{n} R_{i}$ is a partition of $R$ and each $R_{i}$ has property (*) then so too does $R$. This is clear since if this is so then $R$ has property (**) trivially and this has been seen to be equivalent to $(*)$.

Note too that $(*)$ has a compactness property. If for every $\alpha<\beta$ the property $(*)$ holds for each rectangle that projects into the open set $G_{\alpha}$, then $\left(^{*}\right)$ holds for each rectangle $R$ that projects into the open set $\bigcup_{\alpha<\beta} G_{\alpha}$. By compactness, $R$ projects into some finite union $G_{\alpha_{1}} \cup G_{\alpha_{2}} \ldots \cup G_{\alpha_{n}}$ and so $R$ may be partitioned into subrectangles each of which projects into single $G_{\alpha_{i}}$. Since each of these has property (*) it follows, from the additivity property, that $R$ does too.

And finally note that $\left(^{*}\right)$ has a closure property. Let $(a, b)$ be an open interval with endpoints $a, b \in E$. If every rectangle that projects into $(a, b)$ has $\left(^{*}\right)$ then so too does every rectangle $R$ that projects into $[a, b]$. Let such a rectangle $R$ and a gauge $\gamma$ on $\mathbb{N} \times \mathbb{N}$ be given. If an outer corner of $R$ projects to $a$ or $b$ it can be handled as follows. If $R=\left[c_{1}, d_{1}\right] \times\left[c_{2}, d_{2}\right]$ then it may be partitioned into $\left(S_{1}, S_{2}, T_{1}, T_{2}, T_{3}\right)$ where $S_{1}=$ $\left[c_{1}, c_{1}+\epsilon\right] \times\left[c_{2}, c_{2}+\epsilon\right]$ and $S_{2}=\left[d_{1}-\epsilon, d_{1}\right] \times\left[d_{2}-\epsilon, d_{2}\right]$, for arbitrarily small $\epsilon>0$, are two squares and $T_{1}, T_{2}, T_{3}$ are three rectangles which project entirely into $(a, b)$. The subrectangles $T_{i}$ satisfy (**) (iii) (since they project to subsets of $(a, b)$ ) and $S_{1}, S_{2}$ will satisfy (**) (ii) if $\epsilon$ is made sufficiently small since the outer corners project to points in $E$. By definition $R$ must have property (**) (and hence $(*)$ as well) and we are done.

We show now that for each ordinal $\eta$ and any rectangle $R$ that projects to a subset of $G_{\eta}$ the assertion (*) holds. For the initial member of the sequence, $G_{0}=\varnothing$ there is nothing to prove. For $\lambda$, a limit ordinal, the compactness property supplies the statement.

Now suppose that we have verified (*) for all rectangles that project into $G_{\eta}$ and we have a rectangle $R$ that projects into $G_{\eta+1}$. Recall that for some $i$ the set $A_{i}$ is comeager in $G_{\eta+1} \backslash G_{\eta}$. By the additivity property we may assume that $|R|<i^{-1}$.

We apply Corollary 6 with the gauge $\delta^{\prime}$ and with $D=\pi^{-1}\left(G_{\eta}\right)$ and $L^{\prime}=$ $L \cap \pi^{-1}\left(A_{i}\right)$, where $\pi$ is, as before, the projection map $\pi(x, y)=x+y$. By Corollary 6 we may partition $R$ into subrectangles of types $(\alpha),(\beta)$ or $(\gamma)$. The rectangles of type $(\alpha)$ or $(\gamma)$ already have $(*)$ while rectangles of type $(\beta)$ must have $\left(^{*}\right)$ because of the induction hypothesis and the closure property. By the additivity property then $R$ must have property $(*)$ as well and we are done.

The following covering theorem will be the one required for most purposes; here there is no restriction on the gauge. Note that the exceptional set is merely countable.

THEOREM 9. Let $\delta$ be a gauge on $\mathbb{R}$. Then there is a countable set $E$ with the following property: for any 2-interval

$$
K=([a, b],[a+p, b-p]) \quad \text { for } 0<p \leqslant \frac{1}{2}(a+b)
$$

and for any gauge $\gamma$ on $E \times \mathbb{N}$ there is a sequence $\left(I_{1}, I_{2}, \ldots, I_{n}\right)$ forming a 2-partition of the 2-interval $K$ that is $\frac{1}{3}$-regular, such that each $I_{i}$ is either $\delta$-fine at the center or else has an outer endpoint $x$ in $E$ with $\left|I_{i}\right|<\gamma(x, i)$.

Proof. We repeat the arguments used in the proof of Theorem 8 identically, up to the application of Corollary 6 , with the following exceptions. Since the gauge need 
not have the Baire property we are assured only that the sets $A_{i}$ are dense, rather than co-meager, in the portion required; that is, $A_{i}$ is dense (merely) in $G_{\eta+1} \backslash G_{\eta}$. For this proof we let $E=\left\{x_{1}, x_{2}, x_{3}, \ldots\right\}$ be the splattered set of the endpoints of the $\left\{G_{\eta}\right\}$ together with the addition of any countable dense set.

As before we have a rectangle $R$ which projects into $G_{\eta+1}$ and we may assume that $|R|<i^{-1}$. Since $E$ is dense, choose a point $P$ in the rectangle $R$ such that $\pi(P)=$ $x_{j} \in E$. Let $N$ be a neighborhood of $x_{j}$ smaller in length than $\gamma\left(x_{j}, i\right)$ for $i=1,2,3,4$. Use Lemma 3 to partition $R$ into rectangles of type $(\alpha),(\beta)$ or $(\gamma)$ together with up to four rectangles of eccentricity no more than 2 which project into $N$ and have an outer corner projecting to $x_{j}$. These four rectangles are satisfactory for the first four elements of a partition of $R$ by the definition of $N$. The remaining rectangles, since they are of type $(\alpha),(\beta)$ or $(\gamma)$, satisfy $(*)$. Accordingly $R$ satisfies $(* *)$ and hence $\left(^{*}\right)$, as required.

\section{The integral}

Our goal is to introduce an integral which inverts the second order symmetric derivative. Suppose that $F$ is a continuous function for which $\mathrm{SD}_{2} F(x)=f(x)$ everywhere and $f$ is given. How can we recover $F$ ? Equivalently, how can we recover the expression

$$
\Delta_{K_{p}} F=F(a)+F(b)-F(a+p)-F(b-p)
$$

for all 2-intervals $K_{p}=([a, b],[a+p, b-p])$ ? For most applications, however, it is more to the point to recover the expression

$$
\Lambda_{F}(a, b)=\lim _{p \rightarrow 0+} \frac{F(a)-F(a+p)-F(b-p)+F(b)}{p}
$$

which can also be written as

$$
\Lambda_{F}(a, b)=\lim _{p \rightarrow 0+} \frac{\Delta_{K_{p}} F}{p} .
$$

The discussion in Section 2.2 and other considerations lead us to the following definition for the integral. Let $\delta$ be a gauge and let

$$
K_{p}=([a, b],[a+p, b-p])
$$

be a 2-interval. Let us say that a countable set $E$ is an associated exceptional set for $\delta$ if the conclusion of Theorem 9 holds for the pair. Thus for any gauge $\gamma$ on $E \times \mathbb{N}$ there is a 2-partition of $K_{p}$ where each element is $\frac{1}{3}$-regular and each is $\delta$-fine at its center or else has an outer endpoint in $E$ and is $\gamma$-fine at that endpoint.

Definition 10. Let the function $f$ be defined everywhere on an interval $(a, b)$ and let $K_{p}=([a, b],[a+p, b-p])$ be a 2-interval. Then $f$ is said to be $\mathscr{S}^{2}$-integrable on $K_{p}$ with value $I\left(f, K_{p}\right)$ if for every $\epsilon>0$ there is a gauge $\delta$ so that for any associated exceptional set $E=\left\{x_{1}, x_{2}, x_{3}, \ldots\right\}$ there is a gauge $\eta$ on $E \times \mathbb{N}$ such that for any sequence

$$
\left(K_{1}, K_{2}, \ldots, K_{n}\right)
$$

forming a 2-partition of the 2-interval $K_{p}$ that is $\frac{1}{3}$-regular, and such that each $K_{i}$ is either $\delta$-fine at its center or else has an outer endpoint $x_{j}$ in $E$ with $\left|K_{i}\right|<\eta\left(x_{j}, i\right)$ we have

$$
\left|\sum_{i=1}^{n} ' f\left(c\left(K_{i}\right)\right)\left\|K_{i}\right\|-I\left(f, K_{p}\right)\right|<\epsilon
$$

where $\sum^{\prime}$ indicates that the sum is taken only over the $K_{i}$ that are $\delta$-fine at the center. 
It is not immediately clear that the integral is well defined. The main covering theorem, Theorem 9, supplies this.

Lemma 11. Let the function $f$ be defined everywhere on an interval $(a, b)$ and let $K$ be a 2-interval. If $f$ is $\mathscr{S}^{2}$-integrable on $K$ with value $I(f, K)$ then $I(f, K)$ is unique.

Proof. If $I(f, K)=c_{1}$ and $I(f, K)=c_{2}$ and $\epsilon>0$ then, applying the definition, there are two gauges $\delta_{1}$ and $\delta_{2}$ for which the statement of the definition holds. Let $E$ be the countable exceptional set from Theorem 9 using $\delta=\min \left\{\delta_{1}, \delta_{1}\right\}$. Continuing in the definition, there are gauges $\eta_{1}$ and $\eta_{2}$ on $E \times \mathbb{N}$. Let $\eta=\min \left\{\eta_{1}, \eta_{1}\right\}$. Then, by Theorem 9, there exists a sequence $\left(K_{1}, K_{2}, \ldots, K_{n}\right)$ forming a 2-partition of $K$ that is $\frac{1}{3}$-regular, and such that each $K_{i}$ is either $\delta$-fine at its center or else has an outer endpoint $x_{j}$ in $E$ with $\left|K_{i}\right|<\eta\left(x_{j}, i\right)$. This sequence can be used with $c_{1}$ or $c_{2}$ to show that

$$
\left|\sum_{i=1}^{n} ' f\left(c\left(K_{i}\right)\right)\left\|K_{i}\right\|-c_{j}\right|<\epsilon \quad \text { for } \quad j=1,2
$$

and hence that $\left|c_{1}-c_{2}\right|<2 \epsilon$. Since $\epsilon$ is arbitrary, the conclusion follows.

The 'integral' $I\left(f, K_{p}\right)$ taken over the 2 -interval $K_{p}$ may be converted to an ordinary first order integral by the following definition.

Definition 12. A function $f$ defined on an interval $(a, b)$ is said to be $\mathscr{S}$ integrable on $[a, b]$ and we write

$$
(\mathscr{S}) \int_{a}^{b} f(x) d x=\lim _{p \rightarrow 0+} \frac{I(f,([a, b],[a+p, b-p]))}{p}
$$

if this limit exists.

Notice that, in order for the integral $(\mathscr{S}) \int_{a}^{b} f(x) d x$ to be defined, the integral $I\left(f, K_{p}\right)$ must exist for $K_{p}=([a, b],[a+p, b-p])$ and all sufficiently small $p>0$ and the limit in the definition must exist.

The $\mathscr{S}$-integral is easily shown to generalize the Kurzweil-Henstock, or Riemanncomplete integral. It therefore also generalizes the Lebesgue integral and is strong enough to invert ordinary derivatives.

However, as stated in the introduction, our main objective is to invert second symmetric derivatives of continuous functions. The next theorem will present an integrability criterion which will be used to show that the $\mathscr{S}^{2}$-integral realizes this objective. This criterion is also useful for connecting the $\mathscr{S}^{2}$-integral to more familiar Perron type integrals, which are also based on the second symmetric derivative, but we shall not give the details here.

THEOREM 13. A sufficient condition that a function $f$ be $\mathscr{S}^{2}$-integrable on a 2interval $K_{p}=([a, b],[a+p, b-p])$ is that there is a continuous function $G$ on $[a, b]$ so that for every $\epsilon>0$ there is a convex function $H$ with $\Delta_{K_{p}} H<\epsilon$ and there is a gauge $\delta$ such that

$$
\begin{gathered}
\left|G(x+h)+G(x-h)-G(x+k)-G(x-k)-f(x)\left(h^{2}-k^{2}\right)\right| \\
\leqslant H(x+h)+H(x-h)-H(x+k)-H(x-k)
\end{gathered}
$$

for all $x \in(a, b)$ and $0 \leqslant 3 k \leqslant h<\delta(x)$. In that case $I\left(f, K_{p}\right)=\Delta_{K_{p}} G$. 
Proof. Note first that if the inequality of the theorem holds then, writing $K=([x-h, x+h],[x-k, x+k])$, we have

for $0 \leqslant 3 k \leqslant h<\delta(x)$.

$$
\left|\Delta_{K} G-f(x)\|K\|\right| \leqslant \Delta_{K} H
$$

Let $\epsilon>0$ and suppose that $\delta, K_{p}, G$ and $H$ are as in the statement of the theorem. Let $E=\left\{x_{1}, x_{2}, x_{3}, \ldots\right\}$ be any exceptional set associated with $\delta$. Since $G$ is continuous we may select positive numbers $c_{i j}$ so that

$$
\left|\Delta_{J} G\right|<\epsilon 2^{-i-j}
$$

whenever $J$ is a 2-interval with an outer endpoint $x_{j} \in E$ and $|J|<c_{i j}$. Define the gauge $\eta\left(x_{j}, i\right)=c_{i j}$ for each $x_{j} \in E$ and $i \in \mathbb{N}$.

Now consider any sequence $\left(K_{1}, K_{2}, \ldots, K_{n}\right)$ forming a 2-partition of the 2-interval $K_{p}$ that is $\frac{1}{3}$-regular, and such that each $K_{i}$ is either $\delta$-fine at its center or else has an outer endpoint $x_{j}$ in $E$ with $\left|K_{i}\right|<\eta\left(x_{j}, i\right)=c_{i j}$. We use the notation $\Sigma^{\prime}$ to indicate a sum taken over the $K_{i}$ that are $\delta$-fine at the centers and $\Sigma^{\prime \prime}$ to indicate the remaining sum where $K_{i}$ has an outer endpoint $x_{j}$ in $E$. We have then, using (17), (21) and (22), that

$$
\begin{aligned}
\left|\sum_{i=1}^{n} ' f\left(c\left(K_{i}\right)\right)\left\|K_{i}\right\|-\Delta_{K_{p}} G\right| & \leqslant \sum_{i=1}^{n}{ }^{\prime}\left|f\left(c\left(K_{i}\right)\right)\left\|K_{i}\right\|-\Delta_{K_{i}} G\right|+\sum_{i=1}^{n}{ }^{\prime \prime}\left|\Delta_{K_{i}} G\right| \\
& <\sum_{i=1}^{n}{ }^{\prime} \Delta_{K_{i}} H+\epsilon \leqslant \Delta_{K_{p}} H+\epsilon<2 \epsilon .
\end{aligned}
$$

The last inequalities use just the convexity of $H$ (that is, the fact that $\Delta_{K} H \geqslant 0$ for every $K$ ). By definition then, $f$ is $\mathscr{S}^{2}$-integrable on $K_{p}$ and the value of the integral is $\Delta_{K_{p}} G$, as required.

THEOREM 14. Suppose that $G$ is a continuous function on $[a, b]$ and that $K_{p}=([a, b],[a+p, b-p])$ is a 2-interval. If $\mathrm{SD}_{2} G(x)=f(x)$ exists everywhere on $(a, b)$, then $f$ is $\mathscr{S}^{2}$-integrable on $K_{p}$ and

$$
I\left(f, K_{p}\right)=\Delta_{K_{p}} G
$$

Proof. If $\mathrm{SD}_{2} G(x)=f(x)$ exists everywhere on $(a, b)$ then for every $\eta>0$ there is a gauge $\delta$ with

$$
\left|G(x+h)+G(x-h)-2 G(x)-f(x) h^{2}\right|<\eta h^{2}
$$

for $a<x<b$ and $0<h<\delta(x)$. It follows then, using (10) and (11), that

$$
\mid G(x+h)+G(x-h)-G(x+k)-G(x-k))-f(x)\left(h^{2}-k^{2}\right) \mid<\eta C_{1 / 3}\left(h^{2}-k^{2}\right)
$$

for all $x \in(a, b)$ and $0 \leqslant 3 k \leqslant h<\delta(x)$.

Accordingly, we may use $H(x)=\eta C_{1 / 3} x^{2}$, where $\eta C_{1 / 3}=\epsilon$ in the statement of Theorem 13 and the theorem follows.

For the first order integral $(\mathscr{S})$, this theorem takes on the following form.

Corollary 15. Suppose that $G$ is a continuous function on $[a, b]$ such that $\Lambda_{G}(a, b)$ exists and that $\mathrm{SD}_{2} G(x)=f(x)$ exists everywhere on $(a, b)$. Then $f$ is $\mathscr{S}$-integrable on $[a, b]$ and

$$
(\mathscr{S}) \int_{a}^{b} f(x) d x=\Lambda_{G}(a, b)
$$


Proof. This follows directly from (20), Theorem 14 and the definition of the integral.

\section{An application to trigonometric series}

Our main tool in this series is the following classical observation of Riemann. If the trigonometric series

$$
a_{0} / 2+\sum_{n=1}^{\infty} a_{n} \cos n x+b_{n} \sin n x
$$

converges everywhere to a function $f$, then the second formal integral of the series converges to a continuous function $G$, and the second symmetric derivative of $G$ is $f$. From this and Theorem 14 one shows easily that everywhere convergent trigonometric series are $\mathscr{S}$-integrable and may be expressed in Fourier form.

THEOREM 16. Suppose that the trigonometric series (23) converges everywhere to a function $f$. Then $f$ is $\mathscr{S}$-integrable on every period and

$$
a_{n}=\frac{1}{\pi} \int_{c}^{c+2 \pi} f(t) \cos n t d t, \quad b_{n}=\frac{1}{\pi} \int_{c}^{c+2 \pi} f(t) \sin n t d t
$$

in the sense of that integral.

Proof. Let $G$ denote the continuous function represented by the second formal integral of the series. Then, $G(x)-x^{2} a_{0} / 4$ is $2 \pi$-periodic, and since $\mathrm{SD}_{2} G(x)$ exists everywhere, $G$ is smooth. Let $g(x)=x^{2} a_{0} / 4$ and then from (19) we easily compute that

$$
\Lambda_{G}(c, c+2 \pi)=\Lambda_{G-g}(c, c+2 \pi)+\Lambda_{g}(c, c+2 \pi)
$$

for any real $c$. Since $G-g$ is smooth and periodic, the first term on the right is zero, while the second is easily calculated to be $\pi a_{0}$. Since $\mathrm{SD}_{2} G(x)=f(x)$ everywhere, Corollary 15 supplies the correct formula for $a_{0}$, that is, that

as required.

$$
(\mathscr{S}) \int_{c}^{c+2 \pi} f(t) d t=\Lambda_{G}(c, c+2 \pi)=\pi a_{0},
$$

In the same way we obtain a formula for the remaining coefficients; multiply the series $a_{0} / 2+\sum_{n=0}^{\infty} a_{n} \cos n x+b_{n} \sin n x$ by $\cos k x$, use elementary trigonometric identities for $\cos n x \cos k x$ and for $\sin n x \cos k x$, and rearrange the series to obtain a trigonometric series that converges everywhere to $f(x) \cos k x$. For example the term corresponding to $a_{n} \cos k x \cos n x$ is replaced by

$$
a_{n}(\cos (n+k) x+\cos (n-k) x) / 2
$$

before rearrangement. (This is exactly the argument in [10, p. 41] where they point out that it follows from the Rajchman theory of the formal multiplication of trigonometric series.)

As before, the twice formally integrated series converges uniformly and has everywhere $f(x) \cos k x$ for its second symmetric derivative. Consequently (much as before) $f(x) \cos k x$ is integrable and we may integrate it over a period $[c, c+2 \pi]$ for every $c$. Since the constant term in the series for $f(x) \cos k x$ is $a_{k} / 2$ this gives the required formula for $a_{k}$. A similar argument supplies the formula for $b_{k}$. 
This theorem can be expressed directly in terms of our $\mathscr{S}^{2}$-integral over 2intervals, but it assumes a less familiar form (cf. [12, Vol. II, pp. 90-91]).

THEOREM 17. Suppose that the trigonometric series (23) converges everywhere. Then $f$ is $\mathscr{S}^{2}$-integrable on the 2-interval $([-2 \pi, 2 \pi],[0,0])$ and the coefficients may be given by the formulas

$$
a_{n}=\frac{1}{\pi^{2}} I(f(t) \cos n t,([-2 \pi, 2 \pi],[0,0])), \quad b_{n}=\frac{1}{\pi_{2}} I(f(t) \sin n t,([-2 \pi, 2 \pi],[0,0]))
$$

in the sense of that integral.

Other variants and properties of the integrals can be established using standard techniques.

\section{References}

1. J. M. Ash, C. FreILING and D. RinNe, 'Uniqueness of rectangularly convergent trigonometric series', Ann. of Math. 137 (1993) 145-166.

2. A. M. Bruckner, Differentiation of real functions, Lecture Notes in Mathematics 659 (Springer, Berlin, 1978).

3. J. C. BuRkill, Integrals and trigonometric series', Proc. London Math. Soc. 1 (1951) 46-57.

4. A. Denjoy, Leçons sur le calcul des coefficients d'une série trigonométrique (Hermann, Paris, 1941-49).

5. C. Freiling, 'Symmetric derivates, scattered and semi-scattered sets', Trans. American Math. Soc. 318 (1990) 705-720.

6. C. Freiling and D. Rinne, 'A symmetric density property, monotonicity and the approximate symmetric derivative', Proc. Amer. Math. Soc. 104 (1988) 1098-1102.

7. C. Freiling and B. S. Thomson, 'Scattered sets, chains and the Baire category theorem', Real Anal. Exchange 21 (1995-96) 440-458.

8. R. Henstock, The general theory of integration (Oxford University Press, 1991).

9. R. D. JAMES, 'A generalised integral (II)', Canad. J. Math. 2 (1950) 297-306.

10. J. MarcinkiewicZ and A. Zygmund, 'On the differentiability of functions and the summability of trigonometrical series', Fund. Math. 26 (1936) 1-43.

11. D. Preiss and B. S. Thomson, 'The approximate symmetric integral', Canad. J. Math. 41 (1989) $508-555$.

12. A. Zygmund, Trigonometric series (Cambridge University Press, 1968).

C.F. and D.R.

Department of Mathematics

California State University

San Bernardino

California 92407

USA
B.S.T.

Department of Mathematics Simon Fraser University

British Columbia

Canada V5A 1S6 